\title{
Neuropsychological Assessment of Learning and Memory in Rats Following Ketamine Exposure during Late Adolescence
}

\author{
Julianna M. Davis, David Compton* ${ }^{*}$, Miranda Heit, Ashley Fravel, Kimberly Wood \\ Department of Psychology, Behavioral Neuroscience Program, Palm Beach Atlantic University, West Palm Beach, USA \\ Email: ^david_compton@pba.edu
}

How to cite this paper: Davis, J.M., Compton, D., Heit, M., Fravel, A. and Wood, K. (2020) Neuropsychological Assessment of Learning and Memory in Rats Following Ketamine Exposure during Late Adolescence. Journal of Behavioral and Brain Science, 10, 568-589.

https://doi.org/10.4236/jbbs.2020.1012036

Received: November 11, 2020

Accepted: December 20, 2020

Published: December 23, 2020

Copyright $\odot 2020$ by author(s) and Scientific Research Publishing Inc. This work is licensed under the Creative Commons Attribution International License (CC BY 4.0)

http://creativecommons.org/licenses/by/4.0/

\begin{abstract}
With the prevalent issue of drug abuse in society, research regarding the effects of ketamine, a drug frequently abused by youth in club settings, has increased. Despite its potential for misuse, ketamine has demonstrated potential as a fast-acting antidepressant and seems to work well for relieving treatment-resistant depression. However, previous research has shown ketamine use may cause impairments in frontal and medial temporal lobe functioning, leading to problems with memory. While under the influence of ketamine, individuals also display problems with spatial working memory when compared to individuals not dosed with ketamine. The majority of previous research has examined the short-term impact of ketamine use with studies on neurodevelopment largely confined to postnatal exposure. In the present study, the long-term effects on memory caused by repeated ketamine exposure during late adolescence were examined. Rats were used as nonhuman models in order to investigate the cognitive risks resulting from chronic use of ketamine. The results indicated that low-ketamine dosed rats demonstrated significantly better spatial memory recall compared to high-ketamine dosed rats. In addition, high-ketamine dosed rats appeared to struggle more with working memory than the rats in the low-ketamine and control groups. Similarly, both drug groups showed significantly more working memory and reference memory errors than the control group. This indicates that higher doses of ketamine during late adolescence may cause working and spatial memory impairments later in life.
\end{abstract}

\section{Keywords}

Ketamine, Memory Impairments, Long-Term Effects, Spatial Memory, Working Memory 


\section{Introduction}

Ketamine acts as a noncompetitive antagonist at N-methyl-D-aspartate (NMDA) receptors, specifically at the $\mathrm{Ca}^{2+}$ channel pore in the NMDA receptor [1]. NMDA receptors, which are a type of glutamate receptor with excitatory properties, have been linked to the mechanisms of general anesthesia, analgesia, and to neurotoxicity [1]. The anesthetic capability of ketamine has been widely used throughout the medical and veterinary fields [2]. While ketamine was initially used as a battlefield anesthetic, as its popularity increased, the analgesic and dissociative properties of ketamine made it an appealing option for those seeking a drug-induced high [3]. Thus, ketamine became one of many drugs with the potential for abuse.

The use of ketamine can cause distortions in one's perception of auditory and visual stimuli, and higher doses generally cause psychedelic experiences [4]. These properties have caused ketamine to become a popular club drug, resulting in ketamine being classified as a Schedule III drug in the United States [2]. The hallucinatory effects are particularly enticing to younger individuals causing ketamine to be frequently abused in raves and club settings [5].

While there have been very few deaths caused by ketamine overdose, the most common danger of ketamine abuse lies in the drug's ability to leave the user in a confused state. Individuals are more at risk of injury and assault while under ketamine's influence [5] [6]. Thus, while the initial administration of ketamine may not lead to an immediate physical danger, the act of being under the influence of this drug leaves the individual open to environmental threats. Another risk of ketamine use is that individuals who abuse it frequently take it in combination with other illicit drugs like ecstasy, amphetamines, and MDA, which heightens the risk of a hazardous or lethal outcome [5]. Reports of adverse experiences associated with recreational use include memory impairments, a decrease in attention, perseveration and general anxiety, as well as flattened affect and withdrawal, paranoia, ideas of reference, and unusual thoughts [7] [8] [9] [10]. Cardiovascular events such as palpitations and chest pain have also been known to occur with recreational use [11] [12]. Last, while ketamine is a normal injectable, it is now available illicitly in powder and crystal form, in tablets, and as capsules [13].

Along with the danger of abuse, there are other possible risks of ketamine exposure. One concern is the effect ketamine has on developing brains. Ketamine has been frequently used as a pediatric anesthetic which some research indicates may put younger individuals at an undue risk [14]. Research with newborn rhesus monkeys has indicated that there are no significant neurotoxic effects as determined by nerve cell death when the subject was exposed for less than three hours. However, when exposed to ketamine's anesthetic effects for more than nine hours, there was significant nerve cell death in the frontal cortex of the newborn monkeys [14]. This research suggests that extensive exposure to ketamine can cause neural cell death in developing brains. In a similar study, it was found that ketamine might also have neuroprotective effects on the central 
nervous system by preventing inflammation in developing brain when noxious stimuli are present, like the presence of a highly painful stimuli, but has neurotoxic effects when noxious stimuli are absent [15]. Moreover, infants exposed to anesthetic ketamine three or more times had a greater risk of improper neurodevelopment. This promotes the possibility that ketamine can negatively affect brain development and therefore should be used sparingly as a pediatric anesthetic [16]. Last, there are reports of ketamine induced cognitive deficits and neurodegeneration potential to induce the neurodegeneration and cognitive deficits during early development both in vivo as well as in vitro [17] [18] [19] [20].

Despite the possible dangers of ketamine, several studies show that ketamine may have great potential for therapeutic use. After administration, ketamine seems to produce rapid antidepressant effects along with dissociative and psychotogenic effects [21]. Ketamine also shows a strong ability to relieve treatment-resistant depression in patients within hours [2] [22] [23]. Moreover, ketamine aids in the care of treatment-resistant bipolar as well as unipolar depression [23]. Finally, ketamine, as a treatment for depression, exhibits anti-suicidal properties [24].

Another potential therapeutic use for ketamine is as a unique way to treat posttraumatic stress disorder (PTSD). A single infusion of ketamine was sufficient to reduce the symptoms of PTSD [22]. The reduction in PTSD symptoms has been shown to last one to two weeks after ketamine administration with dissociative effects being rare or, if present, transient [25]. While more research is needed, ketamine may provide a viable treatment option for individuals suffering from PTSD.

Since its initial creation in 1962, many researchers have dedicated their time to examining ketamine and its effects. Research has examined the potential for abuse, the various therapeutic aspects, as well as the possible dangerous consequences of using ketamine. However, much of this research has focused on the immediate impacts of ketamine, specifically how ketamine effects an individual while in use or shorty after use. Less research has been dedicated to the long-term effects ketamine might have on an individual's cognitive functioning. Past research indicates that ketamine impairs different aspects of an individual's memory, yet those studies fail to determine if these memory impairments persist. If ketamine is to be used as a treatment for depression, PTSD, or another mental illness, it is vital that all potential negative consequences of ketamine use are fully examined to ensure that the treatment does not cause more harm to the individual. Furthermore, since ketamine is a popular choice among young drug users, it is important to know what lasting effects on cognition ketamine abuse might have [26]. Individuals in late adolescence are not only more susceptible to substance abuse but are also at risk of mental illness [27]. Since ketamine may provide an effective way to treat certain forms of mental illness, it is important to understand what effects ketamine may have on adolescent individuals. Therefore, the purpose of the present study was to examine the long-term effects 
on spatial and nonspatial memory caused by ketamine exposure during late adolescence.

\section{Method}

\subsection{Subjects}

For this study, 26-day old male $(n=21)$ Sprague-Dawley rats, about 84 grams in weight and purchased from Charles River (Wilmington, MA), were used as subjects. These rats were housed in standard $\left(\sim 864 \mathrm{~cm}^{2}\right)$ polycarbonate cages within a climate-controlled facility. The housing facility maintained a 12 -h light/dark cycle, and the rats were provided food (Mazuri Rodent Chow) and water ad libitum. The rats were cared for in coherence with the Guide for the Care and Use of Laboratory Animals [28], under a research protocol approved (11.28.2018) by the Institutional Animal Care and Use Committee of Palm Beach Atlantic University.

Before the beginning of the data collection, one saline rat died due to the stress of transportation. Prior to the start of the study, each rat was randomly assigned to one of three drug treatment conditions: KET $40 \mathrm{mg} / \mathrm{kg}(n=7)$, KET 10 $\mathrm{mg} / \mathrm{kg}(n=7)$, or a saline control group $(n=6)$. Drug exposure began when the rats reached the mid-adolescent period of development (43 days old) and weighed an average of 156 grams. All rats received a total of 10 injections of either $10 \mathrm{mg} / \mathrm{kg}$ or $40 \mathrm{mg} / \mathrm{kg}$ of Ketamine HCL $(100 \mathrm{mg} / \mathrm{ml}$; Henry Schein, Melville, NY) or a corresponding volume of isotonic saline. Intraperitoneal injections were delivered at a volume of $1 \mathrm{ml} / \mathrm{kg}$. During all drug exposure sessions, the ambient temperature was maintained at approximately $24^{\circ} \mathrm{C}$ with the humidity between $45 \%$ and $50 \%$. Drug exposure occurred during postnatal days 43 to 57. Behavioral testing occurred once the rats reached adulthood and had been drug free for approximately 42 days. A timeline of phases of the experiment can be seen in Figure 1.

\subsection{Apparatuses}

\subsubsection{General Activity}

The level of activity for the rats was evaluated in five-minute sessions across two consecutive days in an open field, $60.96 \mathrm{~cm} \times 60.96 \mathrm{~cm}$ chamber consisting of $10.16 \mathrm{~cm}$ squares (i.e., a checkerboard).

\subsubsection{Morris Water Maze}

With the exceptions of general activity and the last two phases of the experiment (see below, WRAM), the assessment of spatial learning and memory included the use of a standard Morris Water Maze (MWM). The MWM utilized for this experiment was a circular white acrylic swimming pool with a diameter of $183 \mathrm{~cm}$ (Figure 2). Different extra-maze cues and escape parameters were used as a function of the task demands associated with the phase of the experiment. The depth of the water was held constant at $30 \mathrm{~cm}$ and was made an opaque white using a nontoxic water-based paint (Sargant Art, Hazelton, 
PA). The MWM was kept in a quiet testing area approximately 7.43 square meters in size. A white curtain served as a barrier from the larger space while the remaining three walls consisted of a neutral beige color, thus limiting the external stimuli available to be used for navigation by the rats during the trials. With the exception of the free swim "probe" trials, a $15 \mathrm{~cm}$ by $15 \mathrm{~cm}$ flat white escape platform was used throughout all phases of assessment in the MWM. For the cued water maze task described later, the escape platform was raised 15 $\mathrm{mm}$ above the surface of the water. For the remaining phases of the MWM assessments, the escape platform was submerged to a depth of $15 \mathrm{~mm}$ below the surface of the water and placed approximately $18 \mathrm{~cm}$ from the wall of the swimming pool.

\subsubsection{Water Radial Arm Maze}

For the last two phases of the experiment, a water-motivated escape version of a standard eight-arm radial maze (Figure 3) was used. This white plastic maze had a height of 24 inches and equally spaced 5.5 -inch by 15.5 -inch arms which could be partitioned off when needed. Once again, the water was made opaque using white nontoxic tempera paint. Square 3.5-inch removable escape platforms which were submerged approximately $15 \mathrm{~mm}$ under the water were used for the trials.

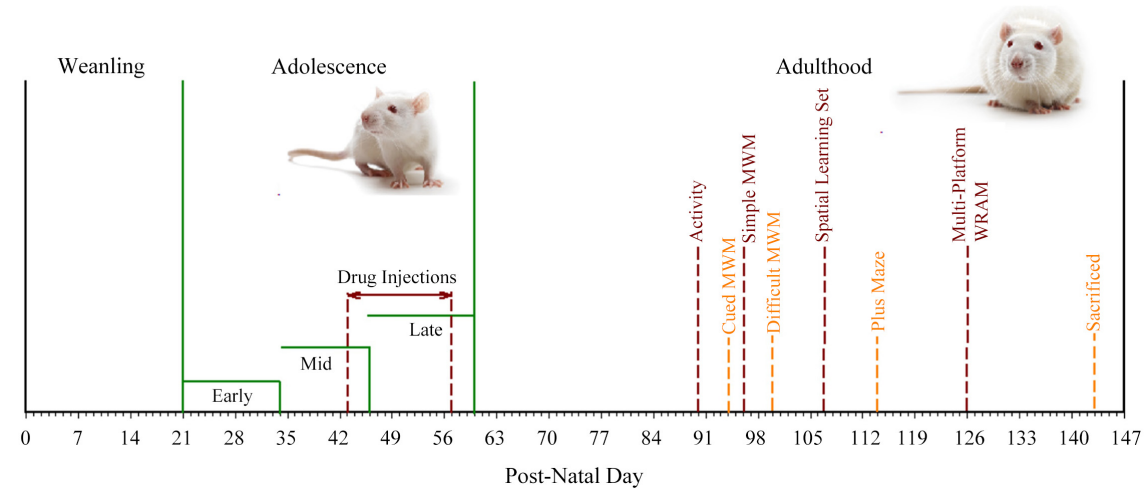

Figure 1. A timeline of the main steps of the study. See Tirelli et al. [29] for additional information on rat adolescence.

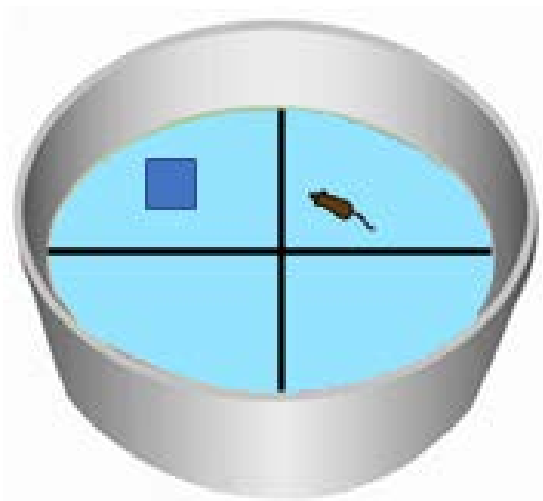

Figure 2. Example of the Morris Water Maze used. 


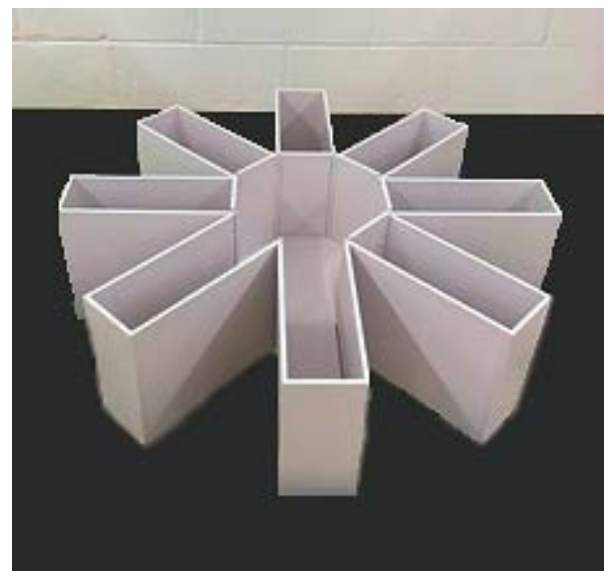

Figure 3. The eight-arm water maze used for the last two phases of the study.

The second-to-last phase of the trial, a nonspatial response learning task, required the WRAM to be made into a simple cross or plus sign configuration by partitioning off the four unneeded arms with Plexiglas barriers.

The last phase of the experiment used the WRAM to simultaneously assess both spatial and working memory using a protocol adapted from Gresack and Frick [30]. Using the full eight arms of the WRAM, escape platforms were placed at the end of four of the arms. One arm was assigned the start arm and never contained an escape platform. The sequence of arms with platforms was determined randomly but remained constant for a given rat during each day of this phase of assessment. In addition, the escape platforms were never located in more than two adjacent arms. A variety of extra-maze cues (e.g., arrows circles, squares) were placed on the walls of the testing room.

\subsection{Procedure}

Our procedure was designed to assess general activity, different facets of spatial and nonspatial learning and memory, as well as response accuracy and difference, if any, in response perseveration.

\subsubsection{Assessment of General Activity}

To measure the general activity level, each rat was evaluated by a lab assistant in five-minute sessions across two consecutive days in the assessment chamber. The dependent measures of activity level included the number of squares crossed and the number of times the rat rose onto its hind legs ("rearing") during the five-minute session.

\subsubsection{Morris Water Maze Tasks}

The series of water maze protocols used here were chosen in order to provide a neuropsychological assessment of rodent learning and memory performance following ketamine exposure without the provision of food deprivation associated with traditional tests of memory. For the first phase of MWM testing, the 
escape platform was visible $15 \mathrm{~mm}$ above the water's surface (cued memory phase); for all remaining phases of MWM testing-the place and spatial learning set phases-the escape platform was submerged $15 \mathrm{~mm}$ below the surface of the water. For all of the spatial assessment phases of the experiment, the rat was gently released into the pool facing the pool's wall at one of the four cardinal compass points, labeled north, south, east, or west, with a designated ceiling of 60 seconds per trial to locate the escape platform. The location of the escape platform varied among one of four compass positions-northeast, northwest, southeast, or southwest. If the rat was unable to locate the escape platform within the designated 60 seconds, it was placed on the platform for 15 to 30 seconds before the next trial commenced. Escape latencies were recorded with a stopwatch, and quadrant crossings, operationally defined as crossing one of the four quadrants associated with the four cardinal compass points, were counted by teams of no fewer than two experimenters.

\section{1) Simple (Cued) Place Learning}

The cued place learning MWM navigation task was administered after the drug recovery period of 42 days and following the assessment of general activity. As a result of drug exposure, a number of sensorimotor and/or motivational changes were possible [31]. Therefore, the cued place learning phase was included to determine whether such non-associative influences developed, especially those that could potentially influence performance on the subsequent place learning, learning set, and MRAM tasks. The cued place learning phase included the use of a platform, readily visible from the surface of the pool, in order to assess general swimming capability, compromised motivation, and nondeclarative memory ability that could influence performance on other phases of the experiment. The rats received 10 trials per day for two consecutive days of testing. On each trial, the escape platform was located in one of four possible locations. Upon successfully locating the platform, the rats were allowed to rest on the platform for approximately 15 seconds at the completion of each trial.

\section{2) Spatial Water Maze Tasks}

Following the cued place learning assessment, the next two phases included an examination of spatial reference memory in the MWM which involved learning the location of a submerged platform that remained constant across all trials within a given phase of the experiment. Although both phases were considered tests of spatial reference memory, they differed in terms of how challenging it was to locate the escape platform. Two variations of the MWM task were used because quite often only minor deficits were found using the standard version of this test [31]. Since the more complex version has been shown to be sensitive for detecting spatial learning and memory impairments following adolescent drug exposure to other drugs such as MDMA or Foxy, it was considered prudent to include it here.

A simple version of the place learning task consisted of training the rats for 10 trials per day for two days in a well-lit environment that included a number of 
extra-maze cues. Between trials, the rats were allowed to remain on the platform for 15 seconds. In addition, a probe trial with the escape platform removed was used as a test of retention on the second day. The probe included testing the subject for a 60 second "free swim", and followed no less than two hours after the last place learning trial. Both the time spent swimming in the target quadrant and the number of crossings over the former platform location were recorded.

The next phase of testing involved a low-cue version of the place learning task and was therefore considered more difficult. Here, the goal was to provide a marked reduction in the availability of cues to aid navigation. In order to accomplish this, the room was indirectly lighted by a single 60 -watt red lightbulb located beyond the curtain, below the horizon of the pool, and approximately three meters from the water maze. As before, a curtain was placed around the water maze allowing few visual cues to aid navigation. For this phase of place learning, the rats were trained for four consecutive trials per day for five consecutive days. As in previous MWM phases, the rats were allowed to remain on the platform for 15 seconds after each trial. Daily probe trials were administered no less than two hours after the last trial of the daily four-trial series.

For the final MWM assessment phase, spatial learning set acquisition, successful escape included the daily requirement that the rat learned a different escape platform location for five consecutive days. All of the animals received four consecutive trials per day. Used as an index of working (short-term) memory, daily trial one and trial two escape latencies and accuracy were compared. Efficient escape latencies on trial two require that the rat recall its prior trial one response. The rats were allowed to sit on the platform for 15 seconds at the completion of each trial.

\subsubsection{Radial Arm Water Maze Tasks}

\section{1) Plus Maze Response Learning}

The first phase in the WRAM and the penultimate phase of the experiment involved the use of plus maze response learning. In this task, successful escape required that the rat learn to choose from three possible response alternatives: to turn left, to turn right, or to swim straight ahead. Using a Fellows series [32], the ordering of placement included two possible starting points. Consistent with all earlier tests, assessment in this phase began by lowering the animal to the surface of the water facing the rear wall of the start arm. As a result, the animal was required to turn $180^{\circ}$ and swim toward the three choices located at the center of the plus maze.

The configuration of requirements was designed to assess both nonspatial response learning as well as perseverative behavior. As noted above, within a given set of trials the animal began at one of two possible starting points. Therefore, the configuration of the available allocentric information differed as a function of each trial. Successful mastery of the task (i.e., learning to turn right vs. left) required that the rat learn a rule to turn in a specific direction regardless of the starting location [33]. The platform remained fixed for each animal until they 
were able to complete nine error-free trials out of the ten daily trials, after which the platform location was reversed. It has been found that the ability to adjust behavior as a function of available allocentric cues is an effective measure of perseverative behavior.

\section{2) Eight Arm Radial Maze}

For the final phase, the full eight arms of the maze were used with one of the arms being designated as the start arm; this start arm never contained an escape platform. The sequence of arms with platforms was randomized but remained unchanged for all sessions in any given day. Platforms were never located in more than two consecutive adjacent arms. At the start of trial one, the rat was released from the start arm and given 90 seconds to locate and climb onto a submerged platform. If the rat did not find a platform within this time, it would be gently guided to the nearest one. Once on the platform, the rat would remain there for 15 seconds, with a 30-second inter-trial interval (ITI). During the ITI, the platform that had been found was removed from the maze, leaving three platforms in the WRAM. The rat would then be returned to the start arm for trial two. This procedure was repeated until all four platforms were located (one platform per trial). Three types of errors were recorded during each trial of the daily sessions. Rats were considered to have entered an arm when the entire body (excluding the tail) entered into the arm. Entries into arms from which a platform had been removed during a daily session were considered working memory errors. In each trial, first entries into arms that never contained a platform were counted as initial reference memory errors. Finally, repeated entries into arms the rat had previously entered were defined as repeated reference memory errors.

\subsection{Assessment of Post-Experiment Brain Glutamate Levels}

Glutamate concentrations in the prefrontal cortex, hippocampus, and striatum were determined as follows. Briefly, the rats from each group were decapitated, followed by removal of the brain from the skull. This step was followed by dissection of the target areas, separated on an ice plate and weighed. The tissue was ground, with $95 \%$ ethanol added. Then, the tissue was centrifuged $(13,000 \times \mathrm{g}$, $20 \mathrm{~min}$ ) at $4^{\circ} \mathrm{C}$. The HPLC analysis included the use of a Shimadzu HPLC system with a UV detector ( $254 \mathrm{nM}$ ) coupled to an LC-10AD Pump, equipped with a C18 analytical column. The mobile phase was consisted of 0.05 M PBS and acetonitrile (Merck; Co, Germany) (70:30 v/v, $\mathrm{pH}=7.2$ ) and was filtered through Millipore prepared $0.45 \mu \mathrm{m}$ durapore membrane filters. The mobile phase was delivered at the flow rate of $0.5 \mathrm{ml} / \mathrm{min}$ and the column temperature was set at $30^{\circ} \mathrm{C}$. Elution of compounds involved a 10 -minute runtime and obtained values were evaluated in $\mu \mathrm{g} / \mathrm{g}$ of the wet tissue.

\subsection{Data Analyses}

For all MWM tasks and the plus maze response learning task, escape latencies 
and navigation errors were the two primary measures of performance. For the WRAM plus maze phase of the experiment, the total of errors was divided into working and reference memory errors. Working, initial reference, and reference memory errors were examined in the final WRAM phase of the experiment.

When exploring the data associated with MWM tasks, depending on the start and escape location, the optimal swim path distances differed considerably. Therefore, the recorded escape latencies for the four start locations were normalized. Normalization involved the computation of the ratio of the minimum swim distance in centimeters for each of the two longer swim paths to the escape platform (e.g., north start location and a southwest goal location) to the minimum swim distance in centimeters of the two shorter swim paths (e.g., a north start location and a northeast goal location [34].

Statistical analyses involved mixed analysis of variance (ANOVAs), with the drug group as the between-subjects factor and days, or blocks of trials and days, as within-subject factors. For WRAM analyses, multivariate analysis of variance (MANOVA) was done with the drug group as the within-subject factor. Post-hoc analyses were performed using paired $t$-tests with a Bonferroni correction or TukeyнsD as appropriate to control for multiple comparisons. The alpha level for acceptance was set at $p<0.05$ and all analyses were performed using SPSS version 23 (2015) [35].

\section{Results}

\subsection{General Activity}

Consideration of the number of rearings and squares crossed across the two days of the assessment period revealed the following. When rearings were considered, no differences associated with the drug were found and the drug $X$ days interaction was nonsignificant. The main effect of day of assessment was significant, $F(1,17)=7.72, p=0.013, \eta_{p}^{2}=0.312$, with animals rearing more on the second day $(M=13.71, S D=6.40)$ than on the first $(M=20.44, S D=11.80)$. Examination of the number of squares traversed revealed no significant effects during the measurement period.

\subsection{Morris Water Maze Assessments}

\subsubsection{Cued Place Learning}

The data associated with cued place learning were collapsed into two blocks of five trials each and assessed for the two days of testing using a 1-between (drug groups), 2-within (days, blocks of trials) ANOVA. Consideration of escape latencies revealed the following. No differences as a function of drug group were found. The main effects of days, $F(1,17)=27.01, p<0.001, \eta_{p}^{2}=0.614$, and blocks, $F(1,17)=10.74, p=0.004, \eta_{p}^{2}=0.387$, were significant, suggesting that escape latencies improved across both blocks of trials and the two days of this phase of testing. None of the interactions were significant (all $p s>0.05$ ).

Assessment of the quadrants crossed revealed a similar pattern with signifi- 
cant main effects of days, $F(1,17)=9.57, p=0.007, \eta_{p}^{2}=0.360$, and blocks, $F(1$, 17) $=25.85, p<0.001, \eta_{p}^{2}=0.603$. Thus, all animals improved within as well as across days. As before, the main effect of drug group and all interactions were nonsignificant.

\subsubsection{Simple Place Learning}

Consideration of escape latency data from the simple (i.e., high available cues) place learning phase revealed similar outcomes. Analysis of the latency data with 1-between (drug groups), 2-within (days, blocks of trials) ANOVA revealed comparable escape latencies across drug groups, with significant within-subject main effects of days, $F(1,17)=15.60, p=0.001, \eta_{p}^{2}=0.479$, and blocks of trials, $F(1,30)=20.22, p<0.001, \eta_{p}^{2}=0.543$. Thus, escape latencies improved as a function of training both within each day and across both days of testing ( $M_{\text {laten- }}$ cies $=4.47, S D=0.789$ vs. $\left.M_{\text {latencies }}=3.76, S D=0.697\right)$. All possible interactions were nonsignificant.

The effects reported above were largely consistent with the analysis of the error data. As was the case for escape latencies, a main effect of days, $F(1,17)=$ 13.02, $p=0.002, \eta_{p}^{2}=0.434$, was found $\left(M_{\text {quadrants }}=4.89, S D=1.58\right.$ vs. $M_{\text {quadrants }}$ $=3.25, S D=1.02)$. However, significant main effects of drug groups and blocks of trials were absent; this was also the case for all possible interactions.

When the platform was removed and the animals tested on a single daily probe trial, escape latencies differed as a function of group, $F(2,17)=3.94, p=$ $0.039, \eta_{p}^{2}=0.317$. Pairwise comparisons revealed that the low-ketamine dose rats spent significantly more time searching the formerly correct quadrant $(M=$ $5.55, S D=0.361)$ than the high-ketamine dose rats $(M=4.87, S D=0.327)$, with the performance of the control animals intermediate and not significantly different from either extreme $(M=5.41, S D=0.676)$. Analysis of the quadrant data yielded similar results.

\subsubsection{Difficult Place Learning}

Although similar to the preceding phase, by using a single 60-watt light bulb located behind the maze curtains, the number of allocentric cues was markedly reduced. For this phase, all trials were normalized, and the four daily trials averaged. Examination of the resulting escape latency data using a 1-between (drug groups), 1-within (days) ANOVA revealed the following. The main effect of drug group approached but was nonetheless nonsignificant, $F(1,17)=2.82, p=$ $0.088, \eta_{p}^{2}=0.249$. Animal performance improved across days of testing, $F(4$, $68)=8.36, p<0.001, \eta_{p}^{2}=330$, but a group $\mathrm{X}$ days interaction was not found. Post hoc examination of the escape latencies supported the main effect finding with escape latencies on days four and five being superior to those on days one and two (Figure 4, Graph A). Analysis of the escape accuracy data was consistent with escape latency data; the animals improved across test days, $F(4,68)=$ 5.98, $p<0.001, \eta_{p}^{2}=0.260$. The pattern of swim-error results largely mirrored that of the escape latency analyses (Figure 4, Graph B). 

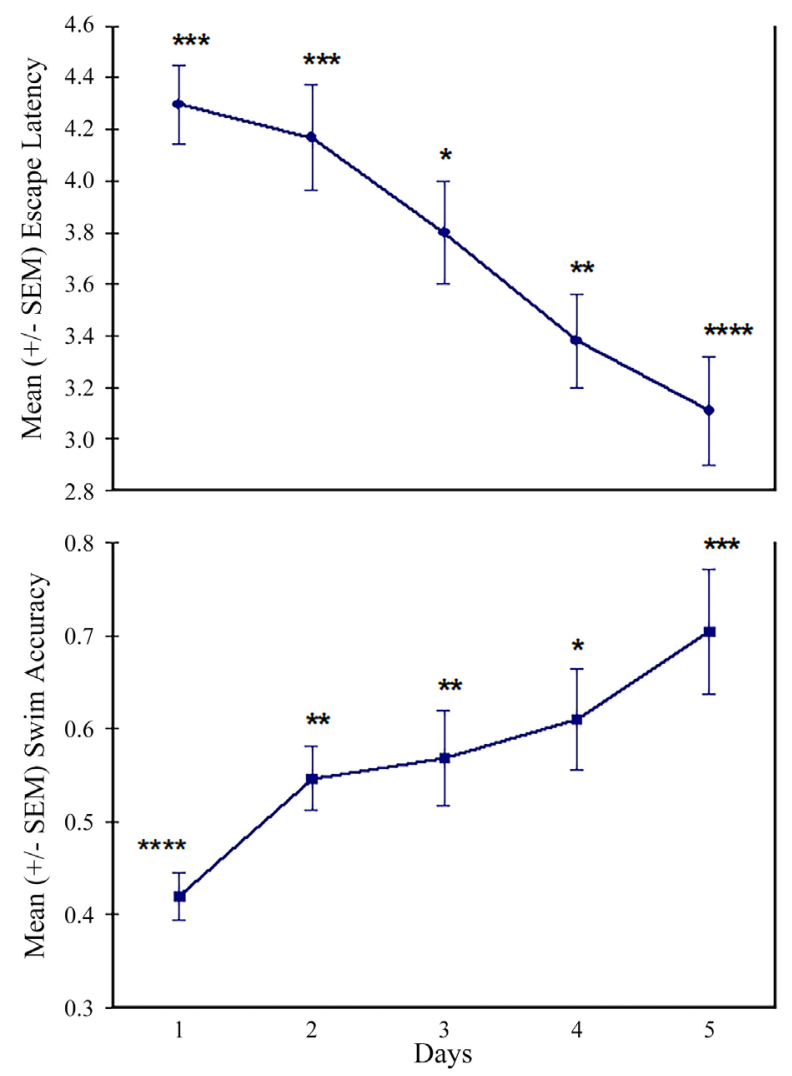

Figure 4. Results of the Hard Place Learning Phase. Panel A: Mean $X=\sqrt{X}$ escape latencies. Pairwise comparisons significantly different. ${ }^{\star * \star \star}$ vs Day $1,2,3,4{ }^{* * \star}$ vs. Day 4 , $5 ;^{\star \star}$ vs. Day 1,$2 ;^{\star}$ vs. Day 5. Panel B: Mean swim accuracy. ${ }^{\star \star * \star}$ vs Day $2,3,4,5 ;^{\star \star \star}$ vs Day $1,2,3{ }^{* *}$ vs Day $1,5{ }^{*}$ vs Day $1 . \mathrm{SEM}=$ standard error of the mean.

\subsubsection{Spatial Learning Set Acquisition Testing}

For each day of spatial learning set testing, trial one versus two performance on the first day was compared to the trial one versus two performance across all five days of testing. Analysis of the resulting data using a 1-between (drug groups), 2-within (days, trials) ANOVA revealed the following. The main effect of group and days were nonsignificant. Conversely, an effect of trial one versus trial two escape latencies was detected, $F(1,68)=33.51, p<0.001, \eta_{p}^{2}=0.663$, reflecting a disruption in escape latencies associated with moving the platform location that resolved in later test days, albeit in an opposite direction. More important, as seen in Figure 5, a group $\mathrm{X}$ days $\mathrm{X}$ trial interaction was found, $F(8,68)=2.82$, $p=0.009, \eta_{p}^{2}=0.249$. Decomposition of the interaction revealed the following findings of interest. On day one of testing, both drug groups had significantly shorter escape latencies on trial two. This was not the case for the control animals. However, the control animals quickly adapted, with lower response latencies on trial two on days two through five. This result mirrored that of the low-ketamine dose group. It was found that escape latencies among the high-dose ketamine group was higher than control animals on trial two of days two through four of testing.

Examination of the search accuracy data produced with some notable excep- 
tions-similar findings (Figure 6). The main effect of drug groups was nonsignificant. On the other hand, the main effect of days was significant, $F(4,68)=$ 2.65, $p=0.040, \eta_{p}^{2}=0.135$, and therefore suggestive of modest changes in navigation accuracy as a function of the number of days of exposure (e.g., $M=$ 0.470 vs. $M=0.564$ ). This result was bolstered by a significant days $\mathrm{X}$ trials interaction, $F(4,68)=8.23, p<0.001, \eta_{p}^{2}=0.326$.

In addition, a group $\mathrm{X}$ days $\mathrm{X}$ trials interaction was found, $F(2,29)=4.93, p<$ $0.05, \eta_{p}^{2}=0.254$, as well as a days $\mathrm{X}$ trials interaction, $F(1,29)=12.10, p<0.05$, $\eta_{p}^{2}=0.294$. Pairwise within-group comparisons revealed changing the platform location initially produced a decrease in search accuracy. However, with the notable exception of the high-dose ketamine animals, by the fourth day of testing response accuracy increased on trial two relative to trial one.

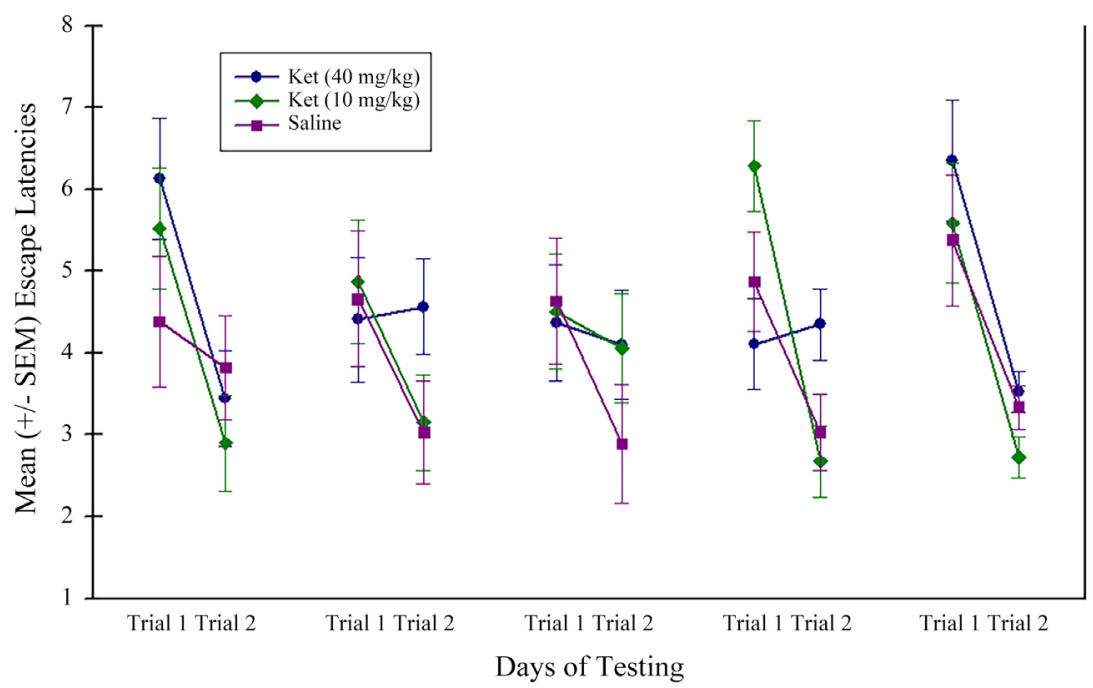

Figure 5. Mean $X=\sqrt{X}$ escape latencies during the spatial learning set phase of testing. $\mathrm{SEM}=$ standard error of the mean.

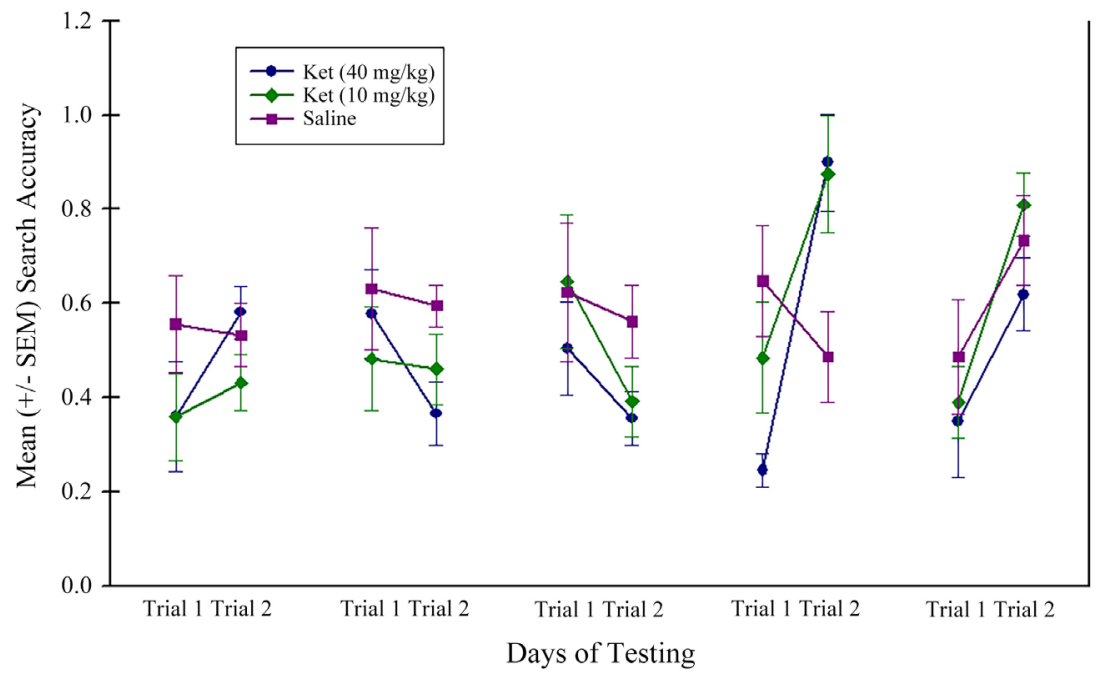

Figure 6. Mean search accuracy during the spatial learning set phase of testing. $\mathrm{SEM}=$ standard error of the mean. 


\subsection{Water Radial Arm Maze Assessments}

\subsubsection{Plus Maze Response Learning}

This phase of testing involved examining various components of nonspatial response learning performance using a plus maze version of the standard MWM. Each day of training consisted of ten trials and continued five days per week for a total of 100 trials. Using an operational framework described elsewhere [23], the total errors were subdivided as either reference or working memory errors. Reference memory errors were scored whenever an animal initially entered one of three incorrect alleys while working memory errors were operationally defined as re-entries into incorrect alleys. Given this, working memory errors can be suggestive of perseverative behavior, leading to longer escape latencies and impaired performance. Thus, in addition to an examination of escape latencies, additional dependent measures included the number reversals, the number of trials to reach the criterion for a reversal (i.e., nine out of ten error-free trials), working memory errors, and reference memory errors. The data were analyzed using a one-way MANOVA, with subsequent univariate $F$ tests if necessary, with weeks serving as a within-subjects factor and the measures of working memory, reference memory, and total errors serving as the dependent measures.

The resulting MANOVA for the effect of drug group was nonsignificant, Wilk's $\lambda=0.558$, approximate $F(8,30)=1.19, p>0.05, \eta_{p}^{2}=0.253$. Perhaps obscured by the marked variability among animals and the modest sample size, given the observed differences between the high-dose ketamine $(M=34.00, S D$ $=9.76)$ and control $(M=47.00, S D=11.54)$ groups, this result may be of interest for further study.

\subsubsection{Eight-Arm Water Maze}

For this phase of the study, working memory errors, reference (i.e., initial error) memory errors, and repeated reference memory errors were jointly considered with the drug group as the independent variable. Thus, as the case for the response learning assessment, the data were examined using a one-way MANOVA. The first day was considered a day of pretraining/practice and the data excluded from analysis. In other words, due to the nature of the task and the novelty of the elements of the protocol, the location of multiple hidden platforms, and, in particular, recognition that the escape platforms disappear once found, the first day did not accurately reflect any working or reference memory domains. Therefore, following the pretraining period that included both maze exposure and the rules of the task, the working and reference memory measures were considered on days two through ten.

The resulting MANOVA revealed a statistically significant difference on the dependent measures as a function of drug group, $F(6,32)=2.52, p=0.043$; Wilk's $\lambda=0.443, \eta_{p}^{2}=0.335$. As seen in Figure 7, subsequent consideration of the univariate ANOVAs revealed a significant effect of drug group on both the working memory $F(2,17)=3.72, p=0.046, \eta_{p}^{2}=0.304$, and initial error $F(2$, 
17) $=4.60, p=0.025, \eta_{p}^{2}=0.351$, measures but not when total reference memory errors were considered. Subsequent pairwise comparisons revealed significant differences in working memory and initial errors between both drug groups and the saline control group. However, no differences between the two drug groups were found.

\subsection{Analysis of Brain Glutamate Levels}

Analysis of glutamate levels in the target brain areas revealed no differences associated with prior ketamine exposure.
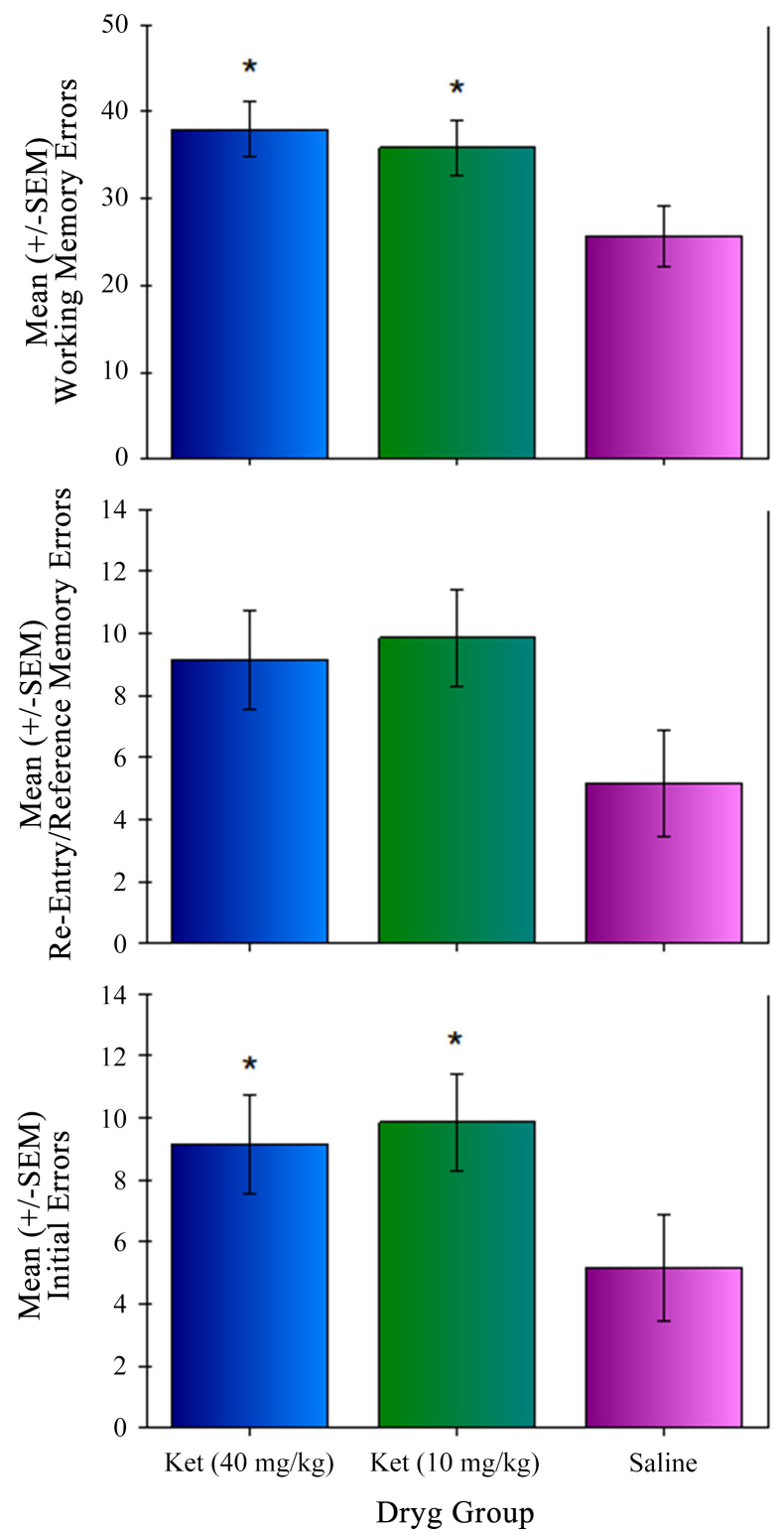

Figure 7. Mean working memory errors, initial error, reference memory errors, and re-entry reference memory errors across the ten days of testing in the WRAM. ${ }^{\star}$ Significantly different $(p<0.05)$ from the saline control rats. $\mathrm{SEM}=$ standard error of the mean. 


\section{Discussion}

In the present study various methods were used in order to determine if ketamine exposure in late adolescence had any effect on spatial and nonspatial learning and memory in adulthood. For most of the Cued Place Learning trials, both the drug groups and the control group improved significantly over time. Similarly, these groups had improved performance over time for both the Simple and Difficult Place Learning with no significant differences among the three groups on the rate of improved performance. The rats' ability to improve their performance across trials indicates that the ketamine administration had no effect on the rats' spatial learning ability, at least in regard to these tasks. The improvement rate between the two ketamine groups and the control group were similar, further suggesting spatial learning was not compromised by ketamine exposure.

During the Simple Place Learning trials, the low-ketamine dosed rats spent significantly more time in the formerly correct quadrant when the platform had been removed for a probe trial than the high-ketamine dosed rats. However, the control rats, which spent more time in the formally correct quadrant than the high-ketamine rats but less time than the low-ketamine rats, did not have any significant difference in performance compared to the drug groups. This would suggest that lower amounts of ketamine have little to no negative effect on spatial memory while higher amounts of ketamine may have a greater long-term effect on an individual's spatial memory. This is interesting as individuals under the influence of ketamine display impaired spatial working memory [36] [37]. Therefore, this impairment may extend beyond the immediate effects of ketamine and could potentially be a lasting effect of the drug.

Despite few significant differences between the ketamine drug groups, and the control group, where found the most noticeable effects of ketamine appeared to be its effects on aspects of spatial reference memory and working memory. The high-ketamine dosed rats exhibited working memory impairments, and both drug groups demonstrated significantly more working and spatial memory impairments than the control group. These results parallel the results of other studies which also found that ketamine, when in use, can cause issues with spatial and working memory [37] [38] [39]. However, the findings of previous research were observed while the subjects were under the effects of ketamine. In the model used for this study, the rats had not been exposed to ketamine for over 42 days; thus, the data suggests potential long-term damage to an individual's working and spatial memory may occur from ketamine abuse in late adolescence. Furthermore, spatial memory and working memory are associated with the prefrontal and parietal cortexes [40]. This might indicate that extended ketamine exposure during late adolescence may cause permanent damage to this area of the brain. Thus, the results of this study coincide with some of the findings of previous studies in regard to spatial and working memory impairments due to ketamine use. However, as this study looked at the long-term effects of 
ketamine, and most previous studies examined the short-term effects, it would seem that some of the spatial and working memory impairments observed during ketamine exposure may persist for long periods of time after the initial exposure.

While some physicians may be wary of using ketamine as a treatment for depression due to the potential for abuse, a study using the olfactory bulbectomy (OBX) model of depression in male rats determined that it is unlikely that the use of ketamine to treat depression will lead to future abuse [41]. After being allowed to self-medicate with ketamine intravenously, the OBX rats did show an increase in ketamine self-administration. However, in the model of relapse, the ketamine-seeking behavior of the OBX rats was shown to be lower than the control animals [41].

In addition to treating depression, ketamine may be useful as a way of treating alcoholism. Preliminary research indicates that those suffering with alcoholism or other addictions can benefit from ketamine administration [42] [43]. One study demonstrated that a single dose of ketamine greatly increased cocainedependent individuals' motivation to quit using cocaine as well as reduced their cravings for the drug. A second ketamine infusion further aided in reducing cue-induced cocaine cravings [44]. Ketamine has been shown to aid recovering heroin addicts and alcoholics in abstaining from their respective drug of abuse [42]. Given that the exact mechanism behind ketamine's ability to potentially treat addiction is unknown, ketamine may enhance neuroplasticity and neurogenesis, provide a treatment for the depressive symptoms, as well as aid the efficiency of psychological therapy [42] [45].

Nonetheless, previous research indicated that ketamine may have some potentially negative effects on neurodevelopment [27]. Since the rats were exposed to repeated doses of ketamine as their brains were still developing, they may have been more susceptible to brain damage resulting in memory impairments which persisted into adulthood. In light of putative treatments that involve the use of ketamine, one thing to consider is that the animals in our study were exposed to ketamine during late adolescence and not in adulthood. If ketamine is to be used as a therapeutic drug in the future, doctors must be careful to ensure that the effects are aiding the patient and not causing them undue harm. The results from this study indicated that spatial and working memory impairments persist later into life if subjects are exposed to repeated doses of ketamine during late adolescence. Unfortunately, individuals during late adolescence are susceptible to both substance abuse as well as mental illness [27]. This should be taken into consideration if ketamine becomes a treatment option for mental illnesses. Using ketamine therapeutically may put these individuals at a greater risk for long-term impairments caused by repeated doses of ketamine.

A limitation of this study was the potential for inaccurate data collection as all data was collected manually through the use of observation, thus the exact number of quadrants crossed or errors made for each rat was left up to the discretion of each pair of observers doing the data collection. Automation of the data collec- 
tion may reduce potential errors. An additional limitation was the small sample size. Future studies should examine long-term effects on memory for subjects exposed to ketamine as adults. Further areas for continued study are to determine if there is a safe level of exposure to ketamine as well as to identify what type of brain damage, if any, is caused by ketamine abuse.

\section{Conclusion}

In summary, in the present study we sought to determine the impact of repeated ketamine exposure during late adolescence on spatial and nonspatial learning memory performance in drug-free adult rats. Ketamine has been shown to be a beneficial way to treat mental illnesses but also has a potential for abuse. In order the timing and dose of ketamine exposure, a rodent model was use to investigate the cognitive risks that may result from chronic use of ketamine during adolescent development. Our results indicated that low-ketamine dosed rats demonstrated significantly better spatial memory recall than rats exposed to a higher dose of ketamine. Further, both drug groups showed significantly more working memory and reference memory errors than the control group. This finding was important for two reasons. First, our results suggest that higher doses of ketamine during late adolescence are associated with working and spatial memory impairments later in life, even when drug free. Second, while adolescent ketamine exposure ultimately did not seem to affect the ability to learn the spatial and nonspatial tasks, the drug did appear to cause lasting impairments to both working and spatial reference memory in our neuropsychological assessments. Therefore, there is a distinct possibility that some of ketamine's effects may not be as transient as initially thought. Further research examining longterm risks of ketamine use should be conducted as well as additional studies examining if the risk of persisting memory impairments is limited to individuals who use ketamine within a certain developmental period.

\section{Acknowledgements}

The authors would like to thank N. Hernandez and B. Gonzalez for their assistance with the assessment of glutamate.

\section{Funding}

This research was supported by a Quality Initiative Grant awarded to David M. Compton

\section{Conflicts of Interest}

The authors declare no conflicts of interest regarding the publication of this paper.

\section{References}

[1] Hirota, K. and Lambert, D.G. (1996) Ketamine: Its Mechanism(S) of Action and 
Unusual Clinical Uses. British Journal of Anaesthesia, 77, 441-444. https://doi.org/10.1093/bja/77.4.441

[2] Garcia-Romeu, A., Kersgaard, B. and Addy, P.H. (2016) Clinical Applications of Hallucinogens: A Review. Experimental and Clinical Psychopharmacology, 24, 229-268. https://doi.org/10.1037/pha0000084

[3] Stone, J.M., Dietrich, C., Edden, R., Mehta, M.A, De Simoni, S., Reed, L.J., Krystal, J.H., Nutt, D. and Barker, G.J. (2012) Ketamine Effects on Brain GABA and Glutamate Levels with 1H-MRS: Relationship to Ketamine-Induced Psychopathology. Molecular Psychiatry, 17, 664-665. https://doi.org/10.1038/mp.2011.171

[4] Wolff, K. and Winstock, A.R. (2006) Ketamine. CNS Drugs, 20, 199-218. https://doi.org/10.2165/00023210-200620030-00003

[5] Dillon, P., Copeland, J. and Jansen, K. (2003) Patterns of Use and Harms Associated with Non-Medical Ketamine Use. Drug and Alcohol Dependence, 69, 23-28. https://doi.org/10.1016/S0376-8716(02)00243-0

[6] Jansen, K.L. (1993) Non-Medical Use of Ketamine. BMJ, 306, 601. https://doi.org/10.1136/bmj.306.6878.601

[7] White, P.F., Way, W.L. and Trevor, A.J. (1982) Ketamine: Its Pharmacology and Therapeutic Uses. Anaesthesiology, 56, 119-136. https://doi.org/10.1097/00000542-198202000-00007

[8] Malhotra, A.K., Pinals, D.A., Weingartner, H., Sirocco, K., Missar, C.D., Pickar, D. and Breier, A. (1996) NMDA Receptor Function and Human Cognition: The Effects of Ketamine in Healthy Volunteers. Neuropsychopharmacology, 16, 120-125. https://doi.org/10.1016/0893-133X(95)00137-3

[9] Newcomber, J.W., Farber, N.B., Jevtovic-Todorovic, V., Selke, G., Melson, A.K., Hershey, T., Craft, S. and Olney, J.W. (1999) Ketamine Induced NMDA Receptor Hypofunction as a Model of Memory Impairment and Psychosis. Neuropsychopharmacology, 20, 106-118. https://doi.org/10.1016/S0893-133X(98)00067-0

[10] Hetem, L.A., Danion, J.M., Diemunsch, P. and Brandt, C. (2001) Effect of a Subanesthetic Dose of Ketamine on Memory and Conscious Awareness in Healthy Volunteers. Psychopharmacology, 22, 59-72. https://doi.org/10.1007/s002130000511

[11] Bowdle, T.A., Radant, A.D., Cowley, D.S., Kharasch, E.D., Strassman, R.J. and RoyByrne, P.P. (1998) Psychedelic Effects of Ketamine in Healthy Volunteers: Relationship to Steady-State Plasma Concentrations. Anaesthesiology, 88, 82-88. https://doi.org/10.1097/00000542-199801000-00015

[12] Weiner, A.L., Vieira, L., McKay, C.A. and Bayer, M.J. (2000) Ketamine Abusers Presenting to the Emergency Department: A Case Series. Journal of Emergency Medicine, 18, 447-451. https://doi.org/10.1016/S0736-4679(00)00162-1

[13] Dotson, J.W., Ackerman, D.L. and West, L.J. (1995) Ketamine Abuse. Journal of Drug Issues, 25, 751-757. https://doi.org/10.1177/002204269502500407

[14] Zou, X., Patterson, T.A., Divine, R.L., Sadovova, N., Zhang, X., Hanig, J.P., Paule, M.G., Slikker Jr., W. and Wang, C. (2009) Prolonged Exposure to Ketamine Increases Neurodegeneration in the Developing Monkey Brain. International Journal of Developmental Neuroscience, 27, 727-731. https://doi.org/10.1016/j.ijdevneu.2009.06.010

[15] Yan, J. and Jiang, H. (2014) Dual Effects of Ketamine: Neurotoxicity versus Neuroprotection in Anesthesia for the Developing Brain. Journal of Neurosurgical Anesthesiology, 26, 155-160. https://doi.org/10.1097/ANA.0000000000000027

[16] Yan, J., Li, Y.R., Zhang, Y., Lu, Y. and Jiang, H. (2014) Repeated Exposure to Anes- 
thetic Ketamine Can Negatively Impact Neurodevelopment in Infants: A Prospective Preliminary Clinical Study. Journal of Child Neurology, 29, 1333-1338. https://doi.org/10.1177/0883073813517508

[17] Campbell, L.L., Tyson, J.A., Stackpole, E.E., Hokenson, K.E., Sherrill, H., McKeon, J.E., Kim, S.A., Edmands, S.D., Suarez, C. and Hall, A.C. (2011) Assessment of General Anesthetic Cytotoxi-City in Murine Cortical Neurones in Dissociated Culture. Toxicology, 283, 1-7. https://doi.org/10.1016/j.tox.2011.01.014

[18] Gomes, L.M., Garcia, J.B., Ribamar, J.S. and Nascimento, A.G. (2011) Neurotoxicity of Subarachnoid Preservative-Free S(+)-Ketamine in Dogs. Pain Physician, 14, 83-90.

[19] Paule, M.G., Li, M., Allen, R.R., Liu, F., Zou, X., Hotchkiss, C., Hanig, J.P., Patterson, T.A., Slikker Jr., W. and Wang, C. (2011) Ketamine Anesthesia during the First Week of Life Can Cause Long-Lasting Cognitive Deficits in Rhesus Monkeys. Neurotoxicology and Teratology, 33, 220-230. https://doi.org/10.1016/j.ntt.2011.01.001

[20] Wang, J., Zhou, M., Wang, X., Yang, X., Wang, M., Zhang, C., Zhou, S. and Tang, N. (2014) Impact of Ketamine on Learning and Memory Function, Neuronal Apoptosis and Its Potential Association with miR-214 and PTEN in Adolescent Rats. PLoS ONE, 9, e99855. https://doi.org/10.1371/journal.pone.0099855

[21] Newport, D.J., Carpenter, L.L., McDonald, W.M., Potash, J.B., Tohen, M. and Nemeroff, C.B. (2015) Ketamine and Other NMDA Antagonists: Early Clinical Trials and Possible Mechanisms in Depression. American Journal of Psychiatry, 172, 950-966. https://doi.org/10.1176/appi.ajp.2015.15040465

[22] Abdallah, C.G., Averill, L.A. and Krystal, J.H. (2015) Ketamine as a Promising Prototype for a New Generation of Rapid-Acting Antidepressants. Annals of the New York Academy of Sciences, 1344, 66. https://doi.org/10.1111/nyas.12718

[23] Abdallah, C.G., Sanacora, G., Duman, R.S. and Krystal, J.H. (2015) Ketamine and Rapid-Acting Antidepressants: A Window into a New Neurobiology for Mood Disorder Therapeutics. Annual Review of Medicine, 66, 509-523. https://doi.org/10.1146/annurev-med-053013-062946

[24] Diazgranados, N., Ibrahim, L., Brutsche, N.E., Newberg, A., Kronstein, P., Khalife, S., Kammerer, W.A., Quezado, Z., Luckenbaugh, D.A., Salvadore, G., Machado-Vieira, R., Manji, H.K. and Zarate Jr., C.A. (2010) A Randomized Add-On Trial of an NMethyl-D-Aspartate Antagonist in Treatment-Resistant Bipolar Depression. Archives of General Psychiatry, 67, 793-802. https://doi.org/10.1001/archgenpsychiatry.2010.90

[25] Liriano, F., Hatten, C. and Schwartz, T.L. (2019) Ketamine as Treatment for PostTraumatic Stress Disorder: A Review. Drugs in Context, 8, Article ID: 212305. https://doi.org/10.7573/dic.212305

[26] Lankenau, S.E. and Sanders, B. (2007) Patterns of Ketamine Use among Young Injection Drug Users. Journal of Psychoactive Drugs, 39, 21-29. https://doi.org/10.1080/02791072.2007.10399861

[27] Schwinn, T.M., Schinke, S.P. and Trent, D.N. (2010) Substance Use among Late Adolescent Urban Youths: Mental Health and Gender Influences. Addictive Behaviors, 35, 30-34. https://doi.org/10.1016/j.addbeh.2009.08.005

[28] National Research Council (2011) Guide for the Care and Use of Laboratory Animals. 8th Edition, National Academies Press (US), Washington DC.

[29] Tirelli, E., Laviola, G. and Adriani, W. (2003) Ontogenesis of Behavioral Sensitization and Conditioned Place Preference Induced by Psychostimulants in Laboratory Rodents. Neuroscience and Biobehavioral Reviews, 27, 163-178. 
https://doi.org/10.1016/S0149-7634(03)00018-6

[30] Gresack, J.E. and Frick, K.M. (2003) Male Mice Exhibit Better Spatial Working and Reference Memory than Females in a Water-Escape Radial Arm Maze Task. Brain Research, 982, 98-107. https://doi.org/10.1016/S0006-8993(03)03000-2

[31] Hartman, R.E., Lee, J.M., Zipfel, G.J. and Wozniak, D.F. (2005) Characterizing Learning Deficits and Hippocampal Neuron Loss Following Transient Global Cerebral Ischemia in Rats. Brain Research, 1043, 48-56. https://doi.org/10.1016/j.brainres.2005.02.030

[32] Fellows, B.J. (1967) Chance Stimulus Sequences for Discrimination Tasks. Psychological Bulletin, 67, 87-92. https://doi.org/10.1037/h0024098

[33] McDaniel, W.F., Via, J.D., Smith, J.S., Wells, D.L., Fu, J.J., Bishop, J.F., Boyd, P.A. and Ledesma, H.M. (1995) Unilateral Injury of Posterior Parietal Cortex and Spatial Learning in Hooded Rats. Behavioural Brain Research, 70, 165-179. https://doi.org/10.1016/0166-4328(95)80006-9

[34] Compton, D.M., Dietrich, K.L., Selinger, M.C. and Testa, E.K. (2011) 5-MethoxyN,N-Di(iso)propyltryptamine Hydrochloride (Foxy)-Induced Cognitive Deficits in Rat After Exposure in Adolescence. Physiology and Behavior, 103, 203-209. https://doi.org/10.1016/j.physbeh.2011.01.021

[35] IBM Corp. (2015) IBM SPSS Statistics for Windows, Version 23.0. IBM Corp., Armonk.

[36] Chan, W., Xu, J., Fan, M., Jiang, Y., Tsui, T.Y., Wai, M.S., Lam, W. and Yew, D. (2012) Downregulation in the Human and Mice Cerebella after Ketamine versus Ketamine plus Ethanol Treatment. Microscopy Research and Technique, 75, 258-264. https://doi.org/10.1002/jemt.21052

[37] Driesen, N.R., McCarthy, G., Bhagwagar, Z., Bloch, M.H., Calhoun, V.D., D’Souza, D.C., Ralitza Gueorguieva, R., He, G., Leung, H.-C., Ramani, R., Anticevic, A., Suckow, R.F., Morgan, P.T. and Krystal, J.H. (2013) The Impact of NMDA Receptor Blockade on Human Working Memory-Related Prefrontal Function and Connectivity. Neuropsychopharmacology, 38, 2613-2622.

https://doi.org/10.1038/npp.2013.170

[38] Curran, H.V. and Morgan, C. (2000) Cognitive, Dissociative and Psychotogenic Effects of Ketamine in Recreational Users on the Night of Drug Use and 3 Days Later. Addiction, 95, 575-590. https://doi.org/10.1046/j.1360-0443.2000.9545759.x

[39] Lofwall, M.R., Griffiths, R.R. and Mintzer, M.Z. (2006) Cognitive and Subjective Acute Dose Effects of Intramuscular Ketamine in Healthy Adults. Experimental and Clinical Psychopharmacology, 14, 439-449.

https://doi.org/10.1037/1064-1297.14.4.439

[40] Passingham, D. and Sakai, K. (2004) The Prefrontal Cortex and Working Memory: Physiology and Brain Imaging. Current Opinion in Neurobiology, 14, 163-168. https://doi.org/10.1016/j.conb.2004.03.003

[41] Babinska, Z. and Ruda-Kucerova, J. (2017) Differential Characteristics of Ketamine Self-Administration in the Olfactory Bulbectomy Model of Depression in Male Rats. Experimental and Clinical Psychopharmacology, 25, 84-93. https://doi.org/10.1037/pha0000106

[42] Ezquerra-Romano, I.I., Lawn, W., Krupitsky, E. and Morgan, C.J.A. (2018) Ketamine for the Treatment of Addiction: Evidence and Potential Mechanisms. Neuropsychopharmacology, 142, 72-82.

https://doi.org/10.1016/j.neuropharm.2018.01.017 
[43] Kolp, E., Friedman, H.L., Young, M.S. and Krupitsky, E. (2006) Ketamine Enhanced Psychotherapy: Preliminary Clinical Observations on Its Effectiveness in Treating Alcoholism. The Humanistic Psychologist, 34, 399-422. https://doi.org/10.1207/s15473333thp3404 7

[44] Dakwar, E., Levin, F., Foltin, R.W., Nunes, E.V. and Hart, C.L. (2014) The Effects of Subanesthetic Ketamine Infusions on Motivation to Quit and Cue-Induced Craving in Cocaine-Dependent Research Volunteers. Biological Psychiatry, 76, 40-46. https://doi.org/10.1016/j.biopsych.2013.08.009

[45] Haile, C.N., Murrough, J.W., Iosifescu, D.V., Chang, L.C., Al Jurdi, R.K., Foulkes, A., Iqbal, S., Mahoney, J.J., De La Garza, R., Charney, D.S., Newton, T.F. and Matthew, S.J. (2014) Plasma Brain Derived Neurotrophic Factor (BDNF) and Response to Ketamine in Treatment-Resistant Depression. International Journal of Neuropsychopharmacology, 17, 331-336. https://doi.org/10.1017/S1461145713001119 